\title{
Collision of a vortex pair with a contaminated free surface
}

\author{
Gretar Tryggvason, Javad Abdollahi-Alibeik, William W. Willmarth, and Amir Hirsa \\ The University of Michigan, Ann Arbor, Michigan 48109
}

(Received 5 December 1990; accepted 25 February 1992)

\begin{abstract}
Collision of a viscous, two-dimensional vortex pair with a contaminated, free surface is studied numerically. The Froude number is assumed to be small, so the surface remains flat. The full Navier-Stokes equations and a conservation equation for the surface contaminant are solved numerically by a finite difference method. The shear stress at the free surface is proportional to the contamination gradient, and simulations for several values of the proportionality constant $(W)$, as well as Reynolds numbers, have been performed. The evolution is also compared with full-slip and no-slip boundaries. As the vortices approach the surface, the upwelling between them pushes the contaminant outward, reducing the amount directly above the vortices, and leading to a clean region for low $W$. As $W$ is increased the clean region becomes smaller, and eventually no clean region is formed. Except for very low $W$, the contaminant layer leads to the creation of secondary vortices, causing the original vortices to rebound in a similar way as vortices colliding with a no-slip boundary. For one case, the numerical results are compared with experimental measurements with satisfactory results. Computations of a vortex pair colliding obliquely with a contaminated surface and head-on collision of axisymmetric vortex rings are also presented.
\end{abstract}

\section{INTRODUCTION}

For fluid mechanics problems involving a free surface, it is often important to account for surface tension effects, particularly for small-scale phenomena. The inclusion of a finite surface tension generally leads to technical complications in both numerical and analytical work. However, even when surface tension is properly accounted for, the results may not account correctly for observations. The reason is, usually, that most fluids are not completely clean, and even a small amount of contaminant can alter the surface tension of a free surface considerably. Modification of waves by the addition of a contaminant and the difference in rise velocity of air bubbles in clean and contaminated water are well-known examples. A simple change in surface tension is, by itself, not a major problem. Rather, the surface flow leads to a nonuniform spatial distribution of contaminants, which, in turn, causes variable surface tension. This nonuniform surface tension can induce surface motion that may alter the flow characteristics considerably. In this paper we study numerically the modification of a simple, unsteady, two-dimensional vortical flow by a contaminated surface.

The evolution of a vortical flow near a free surface and the resulting surface deformation have recently been the subject of several investigations, motivated primarily by a desire to understand the surface signature of ship wakes. Sarpkaya ${ }^{1}$ towed a small delta wing below a free surface, keeping the wing at a negative angle of attack, so that the trailing vortices ascend to the free surface. As the vortices approach the surface, Sarpkaya observed two distinct types of surface signatures. First, before the vortices collide with the surface, relatively irregular and three-dimensional "striations" appear, consisting of streaks perpendicular to the line of motion. These are followed by a pair of long and narrow marks parallel to the line of motion and outboard of the wing. These "scars" appear to be directly related to the trailing vortices, and move outward with the vortices. A different setup, consisting of a two-dimensional vortex pair at low Froude numbers was studied by Willmarth $e t a l^{2}$ and by Sarpkaya $e t$ $a l .^{3}$ for much higher Froude numbers. The surface signature of the pairs is similar to the trailing vortices, but the mean motion is now strictly two dimensional. More complicated flows were studied by Bernal and Madnia, ${ }^{4}$ who investigated the generation of surface waves due to a jet below a free surface and showed that the "opening up" of vortex rings at the surface generates a considerable amount of small-scale surface waves. To explore this mechanism in more detail, Bernal and $\mathrm{K}$ won ${ }^{5}$ experimented with a single ring colliding obliquely with the surface. More recently, Song et al. ${ }^{6}$ have experimented with large vortex rings colliding head on with a free surface.

Several numerical studies of this problem have paralleled the experimental work. Tryggvason ${ }^{7}$ presents a brief numerical study of surface deformation due to the rollup of a submerged vortex sheet using a boundary integral/vortex method, and Willmarth et al. ${ }^{2}$ simulated the formation of a vortex pair from an initially flat vortex sheet and the subsequent vortex and free surface motion. They also made a brief comparison of experimental and computational results. Sarpkaya $e t$ al. $^{3}$ and Telste ${ }^{8}$ also used a boundary integral technique to follow the motion of a point vortex pair toward a free surface. A finite difference simulation of the point vortex problem has been reported by Marcus and Berger, ${ }^{9}$ who also discuss linearized aspects of the problem. A thorough discussion of both vortex collision as well as the formation of vortices from a shear layer and the resulting surface signature, is given by $\mathrm{Yu}$ and Tryggvason, ${ }^{10}$ who simulated a large number of cases and, in particular, explored the limits of high and low Froude numbers. These computations all 
assume an inviscid, two-dimensional motion. Axisymmetric calculations are contained in Song et al. ${ }^{6}$ along with experimental results.

The above experimental and numerical investigations were not concerned with the effects of surface contaminants as such, but it appears that some of the experimental results were influenced by the fact that a free surface is hardly ever completely clean. Earlier, Davies ${ }^{11}$ discussed the damping of turbulent eddies at a free surface (and reviewed earlier work), and Davies and Driscoll ${ }^{12}$ experimented with ejecting pulses of colored water to a free surface, specifically addressing the rate of surface renewal and the effect of contamination on the free surface. They found that the spreading of the colored water at the surface is reduced considerably for contaminated surfaces. However, their primitive visualization technique did not allow for a clear explanation of the mechanism responsible for this behavior. Experiments on the collision of two-dimensional vortex pairs with a free, as well as a rigid, surface were reported by Barker and Crow. ${ }^{13}$ The motivation for their experiments was the observed rebounding of aircraft trailing vortices from rigid (no-slip) surfaces. ${ }^{14}$ This rebounding of a vortex pair from a solid surface is due to the scparation of the ground boundary layer and the subsequent formation of secondary vortices and is therefore not expected if the rigid surface is replaced by a stress-free free surface. However, Barker and $\mathrm{Crow}^{13}$ observed rebounding in their free surface experiments, just as for a rigid surface, and suggested that this rebounding might be due to inviscid effects, such as the deformation of the vortex cores. Saffman ${ }^{15}$ refuted this suggestion and showed that for inviscid flow and a flat boundary, rebounding cannot occur, and suggestcd that the behavior might be due to contamination effects. Peace and Riley ${ }^{16}$ performed numerical simulations of the full Navier-Stokes equations for a plane vortex pair colliding with a flat no-slip and a stress-free surface and argued that even for stress-free boundaries, viscosity could cause rebounding. However, even though their calculations clearly show rebounding, those are for a rather low Reynolds numbers. With an increasing Reynolds number, the rebounding decreased significantly. Their argument can therefore not explain the behavior in the Barker and Crow experiment, which was conducted at a much higher Reynolds number. Recent computations by Orlandi ${ }^{17}$ (as well as those in this paper) show, indeed, that at high enough Re no rebounding takes place for a stress-free boundary.

The explanation for rebounding from a free surface is clear from recent experiments by Bernal et al. ${ }^{18}$ and Hirsa and Willmarth, ${ }^{19}$ who investigated the collision of both vortex rings and planar vortex pairs with a free surface. They observe (as Driscoll and Davis ${ }^{12}$ ) that the cleanliness of the surface influences considerably the vortex motion itself. For a very clean surface, sufficiently weak vortices are deflected outward, in a manner similar to what inviscid theory predicts (if the surface deforms, some rebounding is predicted, but most of the experiments have been performed under conditions where surface deformation is minimal), but for a highly contaminated surface, the behavior was more like vortices encountering a rigid wall. Secondary vorticity from the wall boundary layer is pulled away by the primary vortex that then rebounds as a result of its interaction with the wall vorticity. Detailed observation by laser-induced fluorescence (LIF) led Bernal et al. ${ }^{18}$ to conclude that the surface motion induced by the vortex generates an uneven distribution of surfacc contaminant that, in turn, caused a shear at the surface. The shear generates vorticity that the primary vortex sweeps into the interior, eventually leading to the rebounding of the primary vortex. This injection of vorticity and its subsequent interaction with the primary vorticity appears to be the leading effect of the surface contaminants. Detailed measurements of the velocity field for a vortex pair, as well as observations of the contaminant front motion on the surface for various amounts of contaminant, are presented by Hirsa and Willmarth. ${ }^{19}$

Observations of contaminated surfaces have been reported on numerous occasions for more than a century. One of the more common phenomena is the so-called Reynolds ridge, which appears on the boundary between contaminated and clean surface when the contaminated region is compressed by an inflow of clean water. This is precisely the case when vortices collide with a free surface, as in the experiments of Willmarch and Hirsa. ${ }^{19}$ The upwelling generated by the vortices pushes the contaminated surface water to the side, thereby compressing the contamination layer. The surface above the vortices is clean, and is separated from the contaminated surface by a Reynolds ridge. For a thorough discussion of the Reynolds ridge with historical perspective see Scott. ${ }^{20}$ In the calculations presented here, the surface is assumed to remain flat, so, strictly speaking, no ridge can appear. However, the clean and contaminated surface is often separated by a sharp boundary, and it appears that the vorticity beneath the contaminated surface outboard of this sharp boundary-not the small ridge elevation-is what influences the flow evolution.

The modification of flows with a free surface is of considerable importance in a number of other problems. We mention specifically bubble flow, where contamination sometimes reduces the velocity of a rising buoyant bubble. This effect has been reviewed by Harper, ${ }^{21}$ and recent boundary integral calculations of bubbles in an axisymmetric strain field have been conducted by Stone and Leal. ${ }^{22}$ Flows due to surface tension variations induced by a changing temperature, the Marangoni effect, have been studied on several occasions. ${ }^{23}$

Although rebounding can usually be associated with viscous effects, Dahm et al. ${ }^{24}$ have shown that a weak vortex colliding with a weak density interface can pull up a portion of the interface containing baroclinically generated vorticity that then causes the primary vortex to rebound in completely inviscid simulations. Yu and Tryggvason ${ }^{10}$ also show that a deformable surface can lead to inviscid rebounding. However, this occurs at much higher Froude numbers than in the experiments of Hirsa and Willmarth. ${ }^{19}$ Recent calculations by Ohring and $\mathrm{Lugt}^{25}$ show that rebounding in viscous fluids at high Froude numbers can take place due to secondary vorticity created by large surface curvature. For low Froude numbers their result shows no rebounding, in agreement with experimental observations.

In this paper, wc investigate the modification of a vorti- 
cal flow due to the presence of a contaminant on a free surface. The calculations complement the experiments of Hirsa and Willmarth, ${ }^{19}$ and we compare some of our results to theirs. The fluid is assumed to be viscous, and the full, twodimensional Navier-Stokes equations are solved numerically. Although inviscid methods can easily be modified to account for constant surface tension and can easily predict the redistribution of a surface contaminant, the resulting shear stress is incompatible with the inviscid model. Since we are only concerned with low Froude number flows where surface deformations are small, we assume a flat surface. Threedimensional effects are also assumed to be absent. Preliminary results from similar calculations, but with a more limited scope, have been presented by Wang and Leighton. ${ }^{26}$

In Sec. II we discuss the mathematical model, the numerical method, and the relevant dimensionless parameter. The method is based on a straightforward, second-order, finite difference approximation of the Navier-Stokes equations in vorticity form; hence we give only a brief description. In Sec. III we present our results. First we discuss a number of calculations using a simple constitutive equation for the relation between the surface tension and the contamination and discuss the influence of the relevant parameters; then we compare our results with the experimental study of Hirsa and Willmarth, ${ }^{19}$ using a more realistic constitutive equation. We conclude this section with a brief study of vortex rings colliding head on with the surface and vortex pairs colliding obliquely with the surface. Our conclusions appear in Sec. IV. A short account of some of the work reported here was presented at the Eighteenth Symposium on the Naval Hydrodynamics. ${ }^{27}$

\section{PROBLEM FORMULATION AND NUMERICAL METHOD}

The flow is assumed to be viscous and confined to two dimensions. In addition to two-dimensionality, the major limitation of our calculations is that the free surface is assumed to remain flat for all times. This limits the results presented here to low Froude numbers. However, this is the case that has been most frequently studied experimentally, and since the surface deformations are observed to be small, the limitation is not as severe as one might think. In order to avoid any arbitrary modeling of inflow and outflow boundaries, we simply take the boundary of the domain to be fullslip walls, except for the top. The effects of this limited domain size is discussed in Sec. III.

The flow is governed by the Navier-Stokes equation, which in vorticity form can be written as an advection-diffusion equation for the vorticity,

$$
\frac{\partial \omega}{\partial t}+J(\psi, \omega)=\frac{1}{\operatorname{Re}} \nabla^{2} \omega,
$$

and a Poisson equation relating the streamfunction to the vorticity,

$$
\nabla^{2} \psi=-\omega .
$$

Here $J(\psi, \omega)=(\partial \psi / \partial y)(\partial \omega / \partial x)-(\partial \psi / \partial x)(\partial \omega / \partial y)$, and the Reynolds number is defined as $\operatorname{Re}=\Gamma / v$.
The equations have been nondimensionalized using the initial separation between the vortices and the initial circulation to construct a length and a time scale. Time, velocity, and vorticity are therefore measured in units of $L^{2} / \Gamma, \Gamma / L$, and $\Gamma / L^{2}$, respectively. This gives a Froude number as $\sqrt{\Gamma^{2} / g L^{3}}$, which is small due to the flat surface assumption.

The surface contaminant is assumed to be conserved, leading to a hyperbolic conservation equation

$$
\frac{\partial c}{\partial t}+\frac{\partial}{\partial x}\left(u_{s} c\right)=0
$$

where $u_{s}$ is the horizontal velocity at the surface. Notice that since the surface divergence of $u_{s}$ is, in general, not zero and depends on $c$, this equation allows for the possibility of "contamination shocks" (that is the development of a discontinuity in $c$ ). The surface contaminant affects the flow field through shear stresses induced by variations in the surface tension $\sigma$. This induced shear is given by

$$
\tau=\frac{\partial \sigma}{\partial x} .
$$

Since the surface is flat, the vorticity at the surface is $\omega_{s}=\partial u / \partial y$. The surface tension depends only on the amount of contaminant, $\sigma=\sigma(c)$, and the boundary condition for the vorticity at the surface is therefore

$$
\omega_{s}=\frac{1}{\mu}\left(\frac{d \sigma}{d c}\right) \frac{\partial c}{\partial x} .
$$

If the contaminant is nondimensionalized by its initial value, $c_{0}$, and the vorticity as before, we end up with $\omega_{s}=W \partial c / \partial x$ in nondimensional units, where

$$
W=\frac{L}{\Gamma \mu} c_{0} \frac{d \sigma}{d c} .
$$

The quantity $c_{0}(d \sigma / d c)$ is usually called the elasticity of the surface. The flow is governed by $\operatorname{Re}, W$, and the initial vorticity configuration.

In experiments, the viscosity of the liquid and the elasticity of the contaminant are usually given, and the separation of the vortices may be difficult to change once the experimental apparatus has been built. A change in Reynolds number is thus usually accomplished by an increase in the circulation, $\Gamma$, which, in turn, decreases $W$.

In general, the elasticity of the surface depends strongly on both the composition of the surface contaminant, as well as the amount of contaminant. For most of the calculations reported here, we assume that the elasticity remains a constant. The major reason for this simplification is our desire to focus on the most basic aspect of the problem and to reduce the number of variable parameters as much as possible. For more complicated equations of state, additional parameters are needed. For high $W$, variations in $c$ are generally very small and a linearization around the initial value of the contaminant will give a good approximation. For lower $W$ 's regions of low value of $c$ (even $c=0$ ) appear, but $c$ does not usually increase significantly beyond its initial value (except if $W$ is nearly zero). Therefore, while the linear approximation clearly becomes unphysical at high $c$-predicting negative surface tension - this part of the curve is generally irrelevant, particularly if the initial contaminant concentration is 
low. The computational code is not limited to the simple linear form, and in our comparison with experiments, we have used the appropriate equation of state.

To solve Eqs. (1)-(3) numerically, we use rather standard finite difference approximations. Equation (1) is integrated by an explicit, second-order, predictor-corrector method in time, and the spatial discretization is done with second-order centered differences. For the Jacobian, $J(x, y)$, Arakaw's conservative stencil is used. The Poisson equation, (2), is solved with a fast solver (HWCRT form FISHPAK). For (3) we also use a second-order predictor-corrector in time, and second-order differences in space. The surface velocity is found by a one-sided, second-order differentiation of the streamfunction. For stability, an artificial viscosity term

$$
\frac{\partial}{\partial x}\left[\left(v+a h^{2}\left|\frac{\partial c}{\partial x}\right|\right) \frac{\partial c}{\partial x}\right]
$$

is added on the right-hand side of ( 3 ), with $a$ generally equal to unity. This term is small everywhere except where the contaminant value changes rapidly. At the insistence of a reviewer we have repeated one of our runs using a flux corrected transport method for the contaminant equation. The results obtained were identical to those presented here. For higher viscosities and longer times there would be some differences, naturally, but for the results presented here, a conventional artificial viscosity term is sufficient. Several of our results have been checked for convergence by repeating the calculation using a different resolution.

\section{RESULTS}

\section{A. Parameter studies}

Most of our computations have been done for a vortex pair colliding head on with the top surface. Since the problem is symmetric, it is sufficient to calculate only one of the vortices and use symmetry boundary conditions. Most of the results presented are computed on a $256 \times 256$ grid, but in several cases the calculations have also been done on a coarser $128 \times 128$ grid.

In Fig. 1, we show the evolution of the vorticity for three different boundary conditions on the top surface: (a) is for a stress-free boundary; (b) is for a contaminated surface with $W=0.95$; and in (c) no-slip boundary conditions are enforced. The vortex is initially halfway between the top and bottom boundary, and the vorticity distribution is Gaussian, given by

$$
\omega(x, y)=\left(1 / \delta^{2} \pi\right) e^{\left[\left(x-x_{0}\right)^{2}+\left(y-y_{0}\right)^{2} \mid / \delta^{2}\right.},
$$

where $\delta=0.5$ is in nondimensional units. The computational box is five nondimensional units in each direction. In all cases, the Reynolds number is 1680 . The first frame, at $t=7.4$, is shortly after the motion has started. For the noslip top, a boundary layer has already formed and vorticity is diffusing into the fluid domain. A smaller boundary layer is also visible for the contaminated surface in (b). In the second frame, $t=22.3$, the upward motion of the vortex has ended, and, due to the surface, it is moving outward. The boundary layers in (b) and (c) have grown considerably, and it is clear, in both cases, that separation is about to take place. In the third frame, $t=37.2$, the vortex in (a) contin- ues its outward motion along the full-slip boundaries, but in (b) and (c) the boundary layer has separated and formed a secondary vortex that deflects the path of the primary vortex away from the surface. The strength of the secondary vortex, as well as the rebounding of the primary vortex, is slightly larger for the no-slip surface in (c) than for the contaminated one in (b). This evolution continues in the fourth frame, where $t=52.1$. The vortex in (a) moves out along the top wall, but in (b) and (c), the primary vortex has moved farther away from the top under the influence of the secondary vortex. At the same time, the stronger primary vortex swings the secondary vortex around so it is now almost below the primary one, and thus induces an inward motion. $V$ iscosity has had a visible effect. The maximum vorticity of both the single vortex in (a), as well as both vortices in (b) and (d), has decreased compared with the previous frames. In the last frame, $t=59.5$, the vortex in (a) has encountered the outer boundaries of the computational box and is starting to move downward along the outer wall. In (b) and (c), the primary vortex is moving upward again, as well as inward.

Perhaps the most striking feature of the above sequence is the similarity between the vorticity evolution for the contaminated surface case and the rigid-wall run. In Fig. 2, the vorticity distribution at time 52.1 is shown for $W$ both larger and smaller than in Fig. 1, and same Re. In (a), $W=0.24$; (b) $W=0.48$; (c) $W=4.76$; and (d) $W=23.81$. In the bottom frames, where $W$ is larger than in Fig. 1, the vorticity distribution is virtually indistinguishable from the rigid-wall case. For smaller $W$ in the top frames, vorticity is pulled out from the boundary but not enough to form a secondary vortex. The vorticity "tongue" nevertheless causes the primary vortex to rebound slightly and therefore have lower outward velocity. This effect increases with $W$.

The generation of surface vorticity is directly related to the uneven distribution of the surface contaminant. This distribution is shown in Fig. 3 at times corresponding to those in Fig. 1 for all the runs in Figs. 1 and 2. In (a), the contaminant is passive [corresponding to Fig. 1(a)] and simply advected with the flow, not causing any shear stresses on the fluid at the boundaries. As the vortex collides with the surface, the contaminant is swept outward, depleting the region between and above the vortices of contaminant and accumulating it outboard of the vortices, where the outward velocity decreases. This contaminant peak is then pushed outward. Since the computational box is of a finite width, the outward motion of the contaminant is eventually slowed down by the down-welling at the outer wall of the box. Although the finite box size obviously has effects on the last profiles, the maximum contamination peak increases rapidly, even before the side effects become significant. In the second frame, (b) $W=0.24$, similar evolution is seen, but the rate at which the contaminant is pushed outward is reduced, and the maximum concentration is much smaller. This development continues in (c), where the outward motion has been brought nearly to a halt at the last time due to shear stress created by the uneven contaminant distribution on the top surface. As the vortices rebound, their effect on the surface diminishes, and the contamination "shock" that separates 
(a)
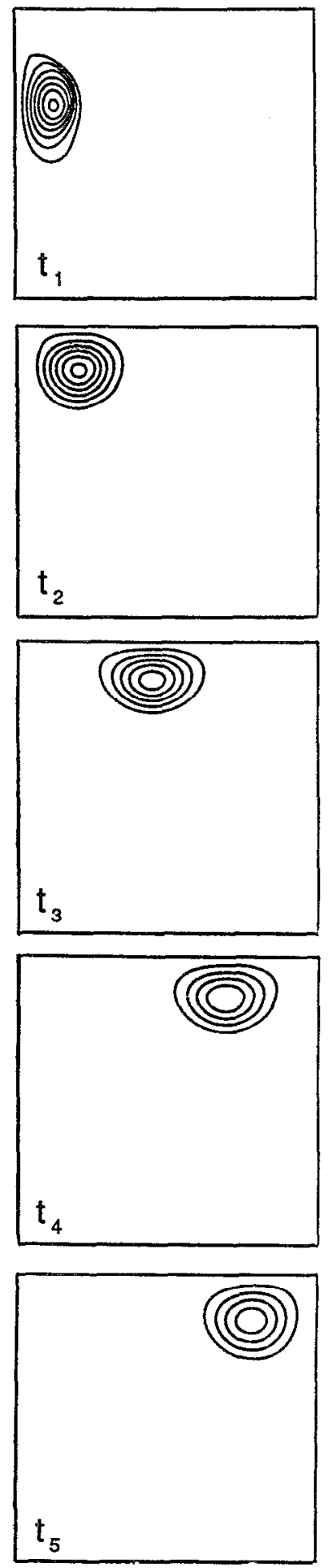

(b)
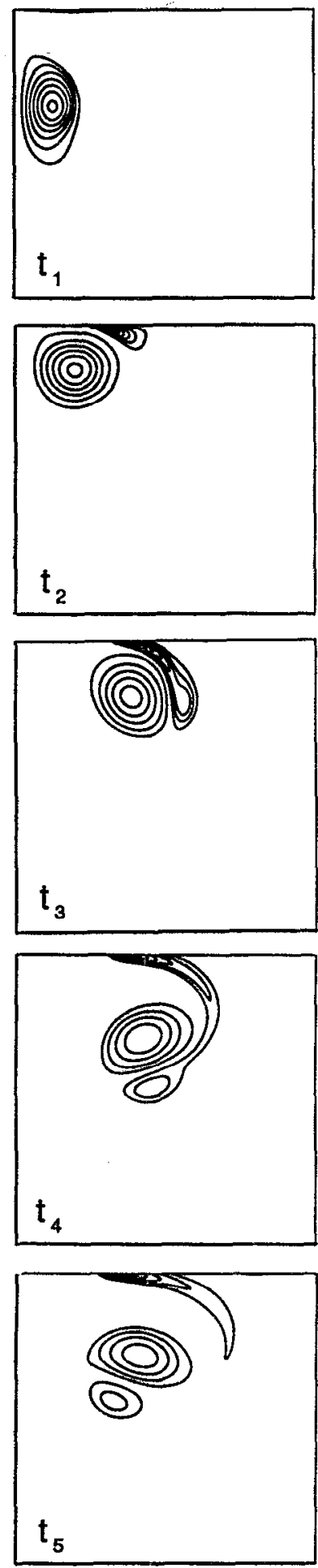

(c)
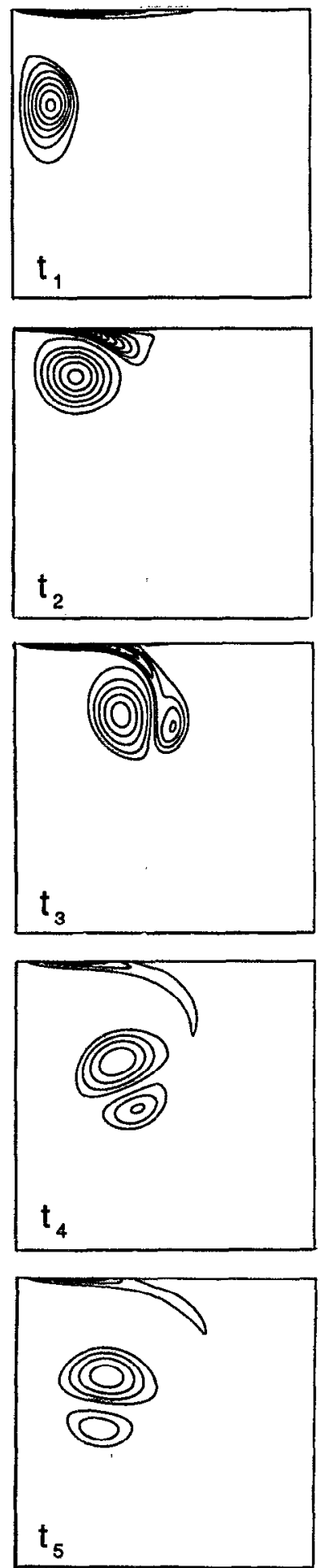

FIG. 1. The collision of a vortex pair with a flat surface. Here $\operatorname{Re}=1680$. The vorticity is shown at times (from the top) $t_{1}=7.4, t_{2}=22.3, t_{3}=37.2$, $t_{4}=52.1$, and $t_{5}=59.5$. (a) Free-slip boundary. (b) Contaminated surface, $W=0.95$. (c) No-slip boundary. 
(a)

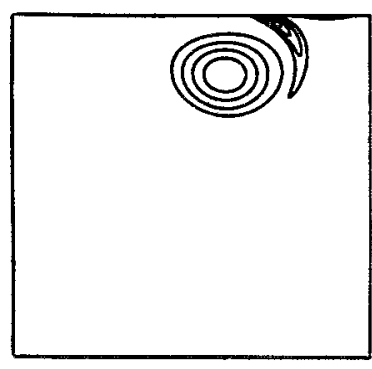

(c)

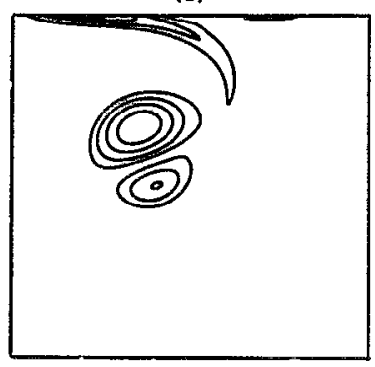

(d)

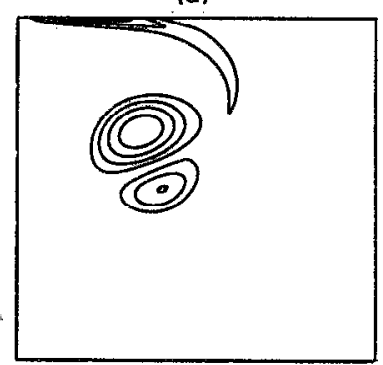

FIG. 2. The vorticity at time $t_{5}=52.1$ for $\operatorname{Re}=1680$ and (a) $W=0.24$; (b) $W=0.48$; (c) $W=4.76$; and (d) $W=23.81$.

the clean and contaminated surface starts to move inward again. In (d), the inward motion has just started at the end of the run. The large accumulation of contaminants, seen in frames (a) and (b), does not take place in (d), and the contamination profile behind the shock equilibrates with time. In frame (e), $W=4.76$, and the restoring effect of the contaminants is much stronger. As a result, only a small clean region forms. The vortices now move behind the shock, and as they pass under and rebound, the "hole" closes rapidly. In (f), $W=23.81$. Here the vortices cause only a small depression in the contamination profile that disappears after the vortices pass by.

To investigate the difference between the results presented above in more detail, we have collected various quantitative measures for both the vorticity and the contaminant distribution. In Fig. 4(a), we plot the path of both the primary and the secondary vortices for the no-stress case, the rigid boundary, and a number of contaminated surfaces. For the full-slip wall, the primary vortex moves outward until it feels the presence of the outer boundary. For the no-slip boundaries, the path of the primary vortex bends away from the surface as soon as a secondary vortex is formed. When the surface is contaminated and $W$ is large, the same rebounding takes place, and for $W=4.76$ and 23.81 , the vortex paths are virtually identical to the solid-wall case. For $W=0.95$, the secondary vortex forms farther outward, and the rebounding is delayed somewhat. A distinct vortex does not form for the lower $W$ cases $(0.24$ and 0.48$)$, but the vortex slows down and rebounds slightly, nevertheless, due to the surface vorticity. (a)

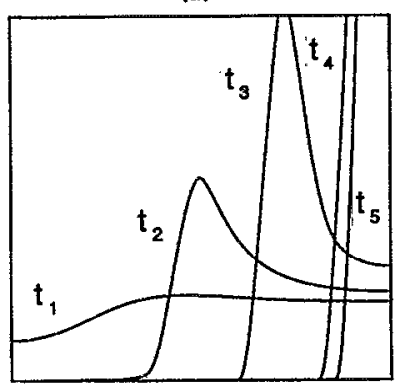

(c)

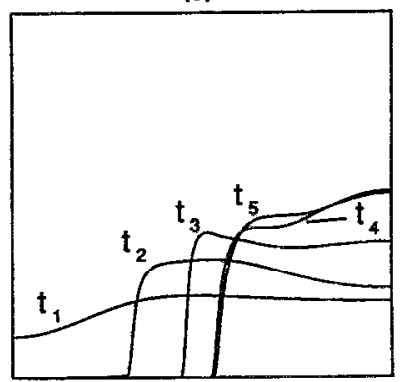

(e)

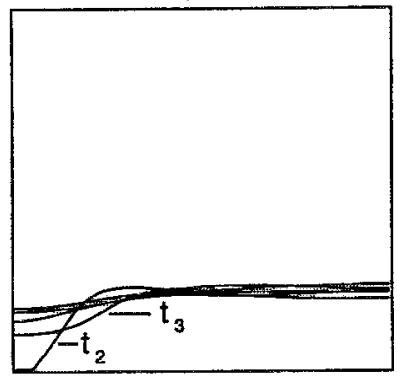

(f)

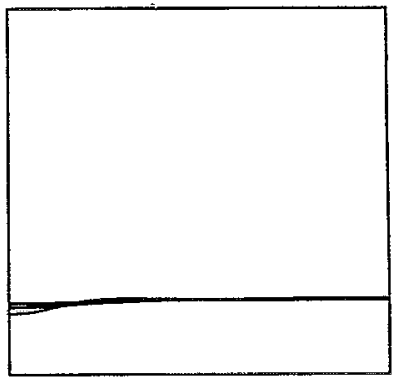

FIG. 3. The contaminant profile at times $t_{1}=7.4, t_{2}=22.3, t_{3}=37.2$, $t_{4}=52.1$, and $t_{5}=59.5$, for the runs in Figs. 1 and 2. (a) Free-slip surface; (b) $W_{1}=0.24$; (c) $W_{2}=0.48$; (d) $W_{3}=0.95$; (e) $W_{4}=4.76$; (f) $W_{\mathrm{s}}=23.81$.

The total vorticity (circulation) of the same sign as the primary vortex is plotted in Fig. 4(b) versus time. When the surface is stress free, the only way to destroy vorticity is by diffusion to the boundaries, which must remain at zero vorticity. For the no-slip and contaminated surface, the top surface not only acquires vorticity of the opposite sign, but this vorticity can also be convected into the fluid domain, thus enhancing mutual destruction of vorticity. This leads to a considerable increase in the rate of loss of primary calculation after the vortices have collided with the top surface. Initially, the calculations with low values of $W$ actually show higher values of circulation than both the no-slip and the stress-free cases. This is due to the generation of vorticity, of the same sign as the primary vortex, at the surface where the contamination profile has a negative gradient. As the surface layer becomes more immobile, this effect disappears. 
(a)

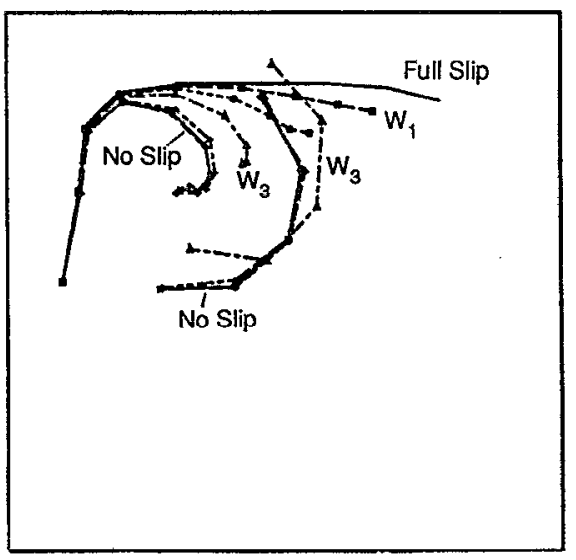

(c)

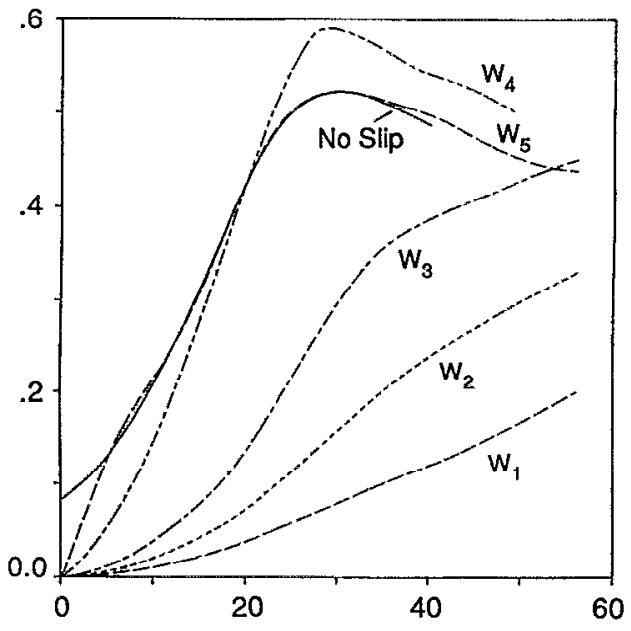

(b)

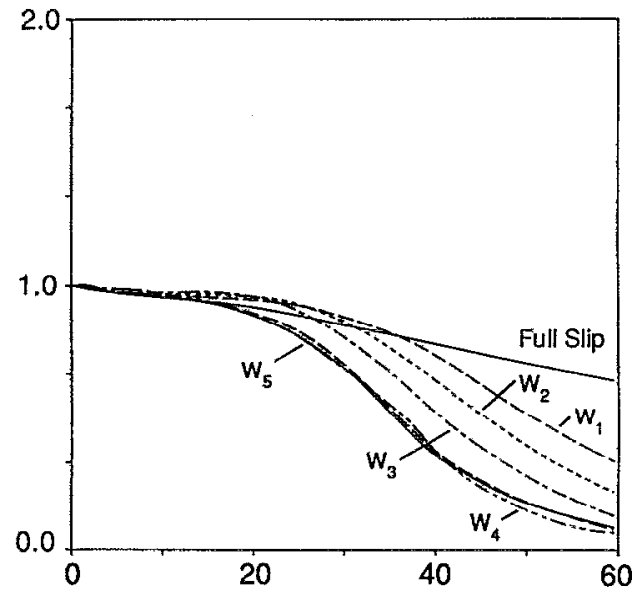

(d)

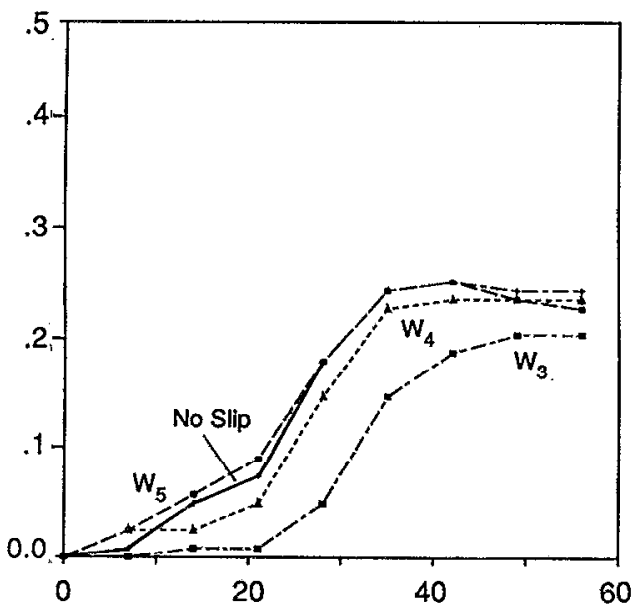

FIG. 4. (a) The path of the vortices for the runs in the previous figures. (b) Vorticity of the same sign as the primary vortex, integrated over the computational domain versus time. (c) Integrated secondary vorticity versus time. (d) Integrated secondary vorticity, excluding a thin layer near the free surface, versus time. ......, full slip; - - , $W_{1}=0.24 ;---, W_{2}=0.48 ;-\cdots--, W_{3}=0.95 ;-\cdots-, W_{4}=4.76 ;---, W_{5}=23.81 ;-$, no slip.

Figure 4(c) is a similar plot, but for the secondary vorticity. As expected, the generation of secondary vorticity is zero for the stress-free boundary and increases with $W$. Since the vortices are initiated relatively close to the surface, there is immediately an outward velocity at the surface. For a noslip surface, vorticity is therefore created instantaneously, but for the contaminated surface, the surface velocity must first redistribute the contaminant, which then, in turn, creates surface vorticity. For the solid-wall case and the higher $W$ 's, the primary vortex eventually rebounds, and as the vortex pair moves away from the boundary, the generation of secondary vorticity is greatly reduced. Due to diffusion, secondary vorticity also undergoes mutual destruction with the primary vorticity. $\Lambda$ somewhat surprising feature of the graph in Fig. 4(c) is that the curves do not approach the solid-wall case monotonically. In particular, the $W=4.76$ case actually has more secondary vorticity at late times than do the $W=23.81$ and the solid-wall cases. The explanation for this is that when there is significant variations in the contamination profile, as is the case for $W=4.76$, vorticity generation does not cease as the vortices move away from the boundary, as in the no-slip case. The surface vorticity is still proportional to the contamination gradient, and remains nonzero until the contamination profile is flat again. For a given contamination gradient the vorticity increases with $W$, but for $W=23.81$ the contamination profile remained nearly flat for all time, so even though $W$ is higher, variations in $c$ are smaller. To separate the surface vorticity from the vorticity of the secondary vortex, we have.estimated its strength by excluding a layer near the surface when we integrate the secondary vorticity [Fig. 4(d) ]. For $W=4.76$ and 23.81 , as well as the solid-wall case, this gives the strength of the secondary vortex about one-fourth of the strength of the primary one, once the vortices have rebounded. For $W=0.95$, 
the strength is one-fifth of the primary vortex.

The above comparisons have focused on the vorticity distribution. To quantify the evolution of the contamination profile in a simple way, we plot in Fig. 5(a) the second moment of the profile. If the profile remains flat, as for high $W$ 's, this quantity remains zero; if the "hole" continues to grow, as for the stress-free surface, it increases constantly. For $W$ high enough so that the outward motion of the contaminant stops, the curve levels off, eventually bending down again when the profile starts to become flat again. As the graph shows, the profile for $W=0.24$ is still changing at the end of the run, although at a considcrably lower rate than for the stress-free boundary. In all other cases, the growth has stopped and is actually slightly negative at the end of the

(a)

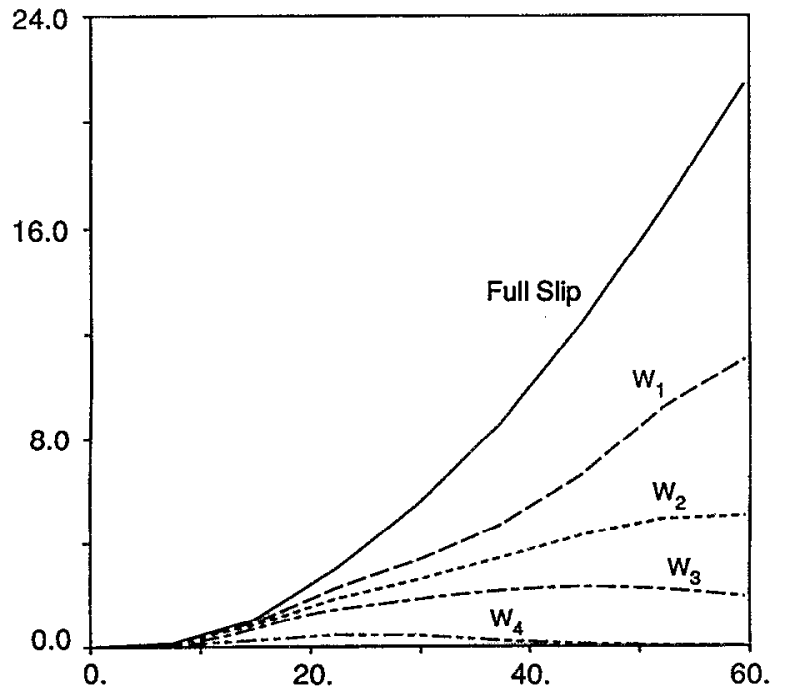

(b)

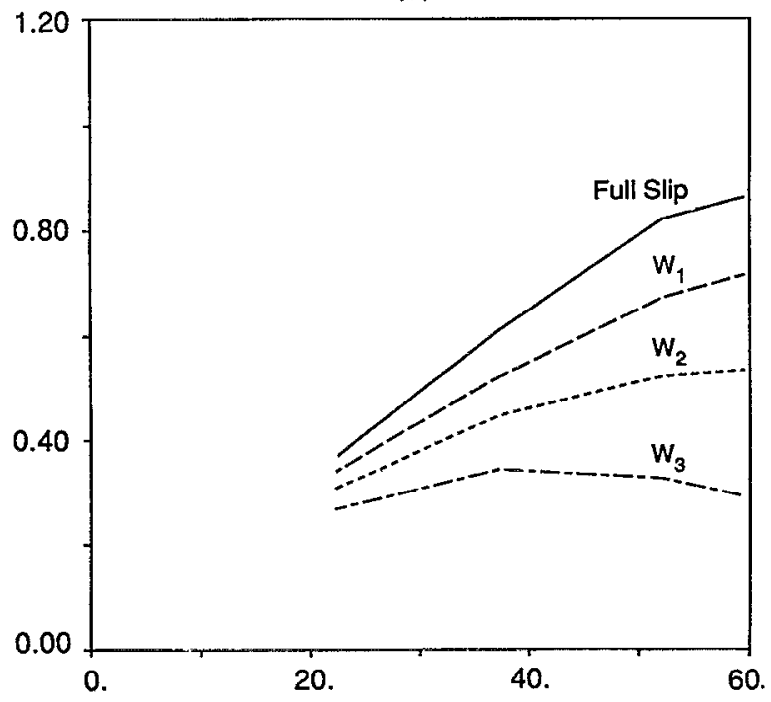

FIG. 5. (a) The second moment of the contamination profile $f\left(c-c_{0}\right)^{2} d x$ versus time for the runs in the previous figures. (b) The position of the contaminant jump versus time for the cases where a clean "hole" forms. run. In Fig. 5(b), we plot the position of the contamination jump, for those cases and those times where it has formed. Since the jump is not completely sharp (a slight artificial viscosity is used to prevent oscillations), we determine the position simply as the point where the profile crosses a horizontal line at half the initial concentration. The result shows what has been pointed out before, that the outward motion stops for sufficiently high $W$, and the "hole" closes again at the end of the large $W$ runs.

The above calculations have all been done in a relatively small computational domain. To assess the influence of the boundaries on the evolution, we have repeated a few of the runs in a domain that is twice as wide. In Fig. 6, we show the vorticity, as well as the contamination profiles, for $W=0.95$ at time 52.1 for both the short and the long computational domains. The vorticity distribution is obviously almost identical, but the value of the contamination concentration is slightly higher behind the shock for the shorter box, and, as a result, the shock moves slightly faster to close the hole in the contamination profile after the vortices have rebounded. At earlier times the differences are even less, and other cases show similar agreement. Even for a stress-free boundary there is good agreement at early times, although at late time there are differences, since no rebounding takes place and the vortex continues to move outward in the longer box. These tests suggest that boundary effects are minimal for the results we show, particularly for those cases where rebounding takes place. We have also checked the effect of the initial depth of the vortex, and except that the initial deformation of the contamination profile is slower, no significant differences arise. In particular, the maximum opening of the contaminant "hole" and the path of the vortices remain essentially unchanged. (For low Reynolds numbers, diffusion has a longer time to modify the vorticity distribution; at higher $\mathrm{Re}$, this effect is insignificant.)

All the calculations presented so far have been at a Reynolds number of 1680 . This number reflects a compromise between our desire to consider as high $\mathrm{Re}$ as possible (since experiments are usually carried out at considerably
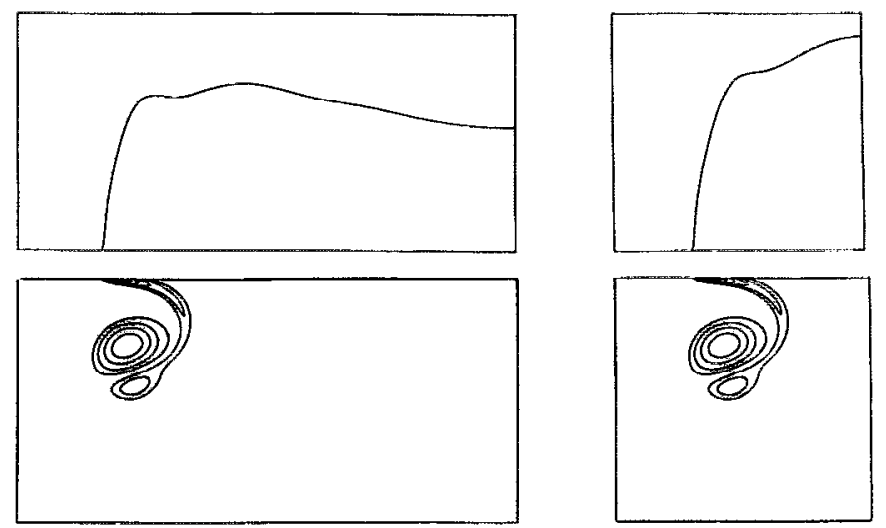

FIG. 6. The solution for $\operatorname{Re}=1680$ and $W=0.95$ at time 52.1 , as calculated both in a short and long computational domain. 
(a)
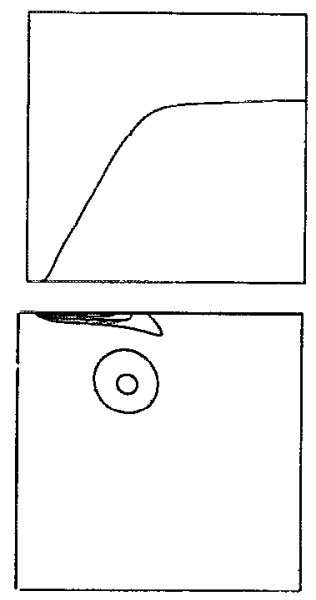

(b)
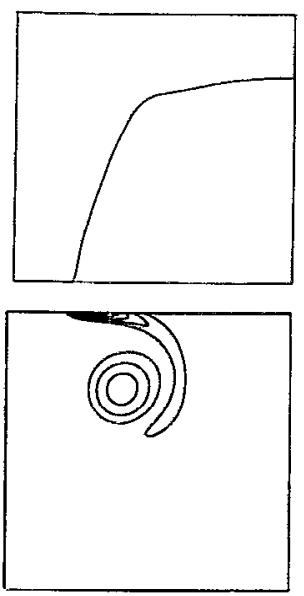

(c)
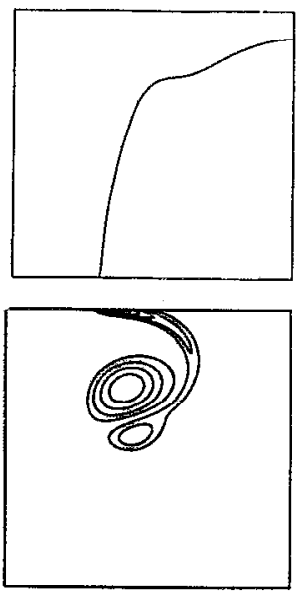

(d)
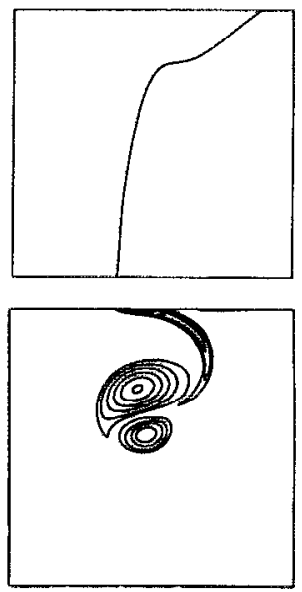

FIG. 7. The effect of Reynolds number. (a) $\operatorname{Re}_{1}=420$; (b) $\operatorname{Re}_{2}=840$; (c) $\operatorname{Re}_{3}=1680$; (d) $\operatorname{Re}_{4}=3360$. The vorticity and the contamination profiles at time 52.1 .

higher $\mathrm{Re}$ ), and the increased resolution requirement, and thus computer time, for high Re calculations.

To explore the influence of the Reynolds number, we have compared calculations with $W=0.95$ and $R e=420$, 840 , and 3360 , with the results from Figs. 1 (b) and 3(d) $(\mathrm{Re}=1680)$. The vorticity distribution and the contamination profile at time 52.1 are shown in Fig. 7. Obviously, the vortices diffuse faster the smaller the Reynolds number. In Fig. 7 (a) $(\operatorname{Re}=420)$, the primary vortex has almost fully disappeared, and no secondary vortex is visible. In Fig. 7(b) $(\operatorname{Re}=840)$, there is only a small "tongue" of secondary vorticity, whereas in Fig. 7(c) $(R e=1680)$, a distinct secondary vortex has formed. For the highest Reynolds number in Fig. $7(d)(R e=3360)$, this secondary vortex is even stronger. The change in vorticity is reflected in the contamination profiles. In all cases, a clean region is generated above the vortex center, but the maximum width of this region increases with Reynolds number, and the reclosing is delayed.
In Fig. 8(a), the trace of the position of the maximum vorticity is shown. For low $\mathrm{Re}$, no secondary vortices form, and the primary vortex slows down rapidly as its circulation diminishes. Since vorticity diffuses rapidly toward the top surface, the position of maximum vorticity does not reach as close to the surface as the stronger vortices at higher Reynolds number do, and actually an apparent rebounding, due to this effect, takes place at the end of the run. The high $\mathrm{Re}$ vortices first move outward parallel to the free surface, but then take a rather sharp downward turn as the secondary vortex appears. The effect of vorticity diffusion is clear in Fig. 8(b), where the integral of all vorticity of the same sign as the primary vorticity is plotted versus time. For all cases, the rate of diffusion changes significantly once secondary vorticity of opposite sign has been generated. For the highest Reynolds number, there is actually a slight increase in the total vorticity initially; this is due to negative vorticity generated at the surface, where the contaminant profile exhibits a negative gradient. Such negative vorticity is also generated (a)

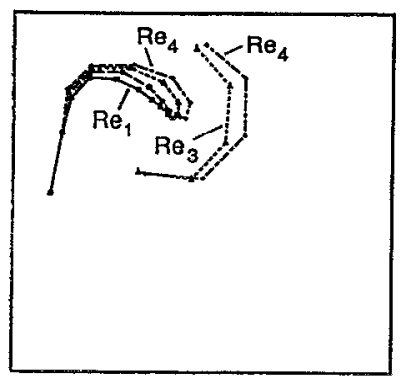

(b)

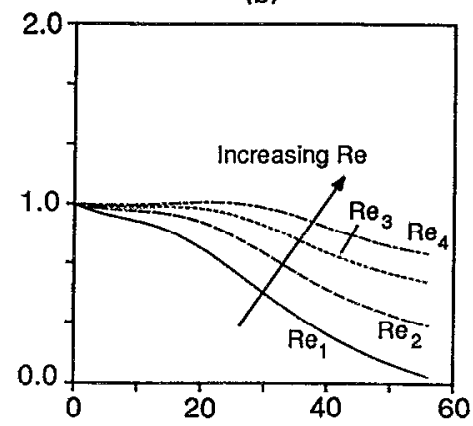

(c)

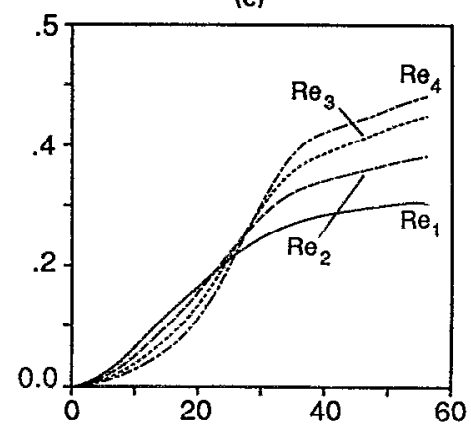

FIG. 8. The effect of Reynolds number. Notation as in Fig. 7. (a) The path of the vortices for the runs in the previous figure. (b) Vorticity of the same sign as the primary vortex, integrated over the computational domain versus time. (c) Integrated secondary vorticity versus time. 
for lower Re, but does not contribute enough to increase the total amount. The growth of the secondary vorticity, Fig. $8(\mathrm{c})$, is initially largest for low Re, where the surface vorticity diffuses most rapidly into the domain. However, as the strength of the primary vortex is reduced, the rate at which secondary vorticity is produced decreases. Although little secondary vorticity is created initially for the high Reynolds numbers, the production increases rapidly as separation takes place. As the primary and secondary vortex rebound, the rate at which secondary vorticity is produced decreases again. The Reynolds number effects on the contamination profiles are quantified in Fig. 9, where the second moment is plotted versus time. In all cases, there is a considerable growth in this quantity, reflecting that the distribution forms a clean hole. The variations are smallest for the low Reynolds numbers and start first to decline for those cases, since the contamination begins to equilibrate first there. For low Reynolds numbers, diffusion is more effective than rebounding in reducing the influence of the vortices on the surface contaminant. Up to about time 20 , there is relatively small difference in the contamination for the largest Re.

Notice that the comparison presented in Figs. 7-9 is not directly representative of an experimental condition, where the Reynolds number is increased by either increasing the strength of the vortices or decreasing the viscosity of the liquid (by using different liquids). Since both $\Gamma$ and $\mu$ enter into $W$, it would generally change as well, whereas here we keep $W$ constant. A comparison for such a situation, where $W$ changes as a result of change in viscosity, is given by comparing Fig. 7(d) and Fig. 2(b) [and Fig. 3(c)]. In the calculations in Fig. 2(b) relatively little secondary vorticity forms, and the "hole" in the contamination profile continues to grow. Obviously there is much less similarity between these cases than the cases shown in Figs. 7(c) and 7(d). Changing $\Gamma$, while keeping everything else constant, corresponds to comparing the $\operatorname{Re}=3360, W=0.95$ run to

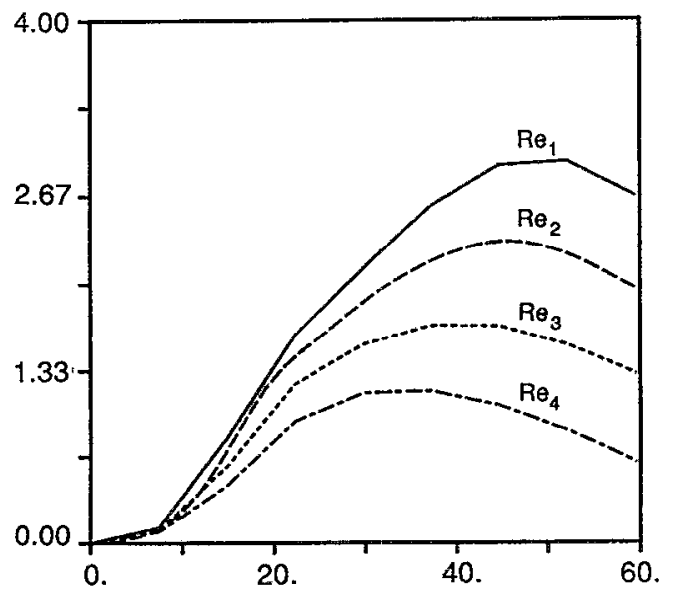

FIG. 9. The second moment of the contamination profile, $\int\left(c-c_{0}\right)^{2} d x$ versus time, for the runs in Fig. 7. Notations are as in Fig. 7. (a)
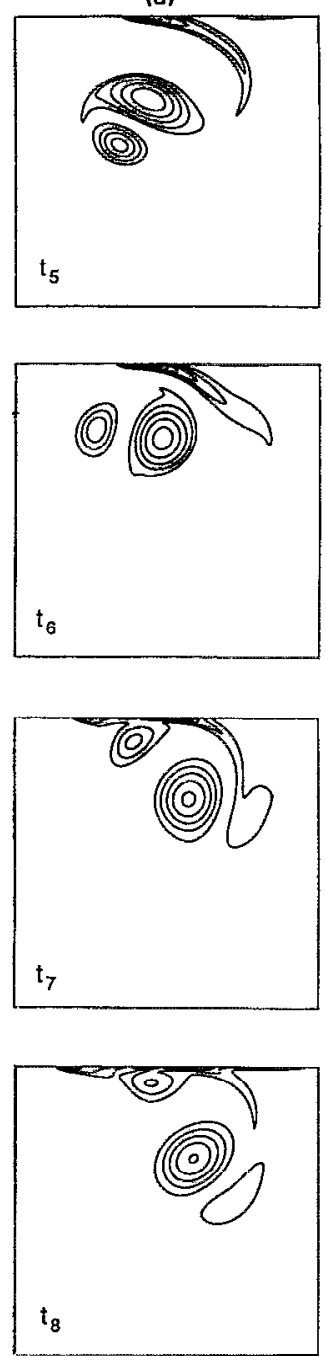

FIG. 10. Long time evolution for $\operatorname{Re}=3360$. (a) The vorticity at various times. Here $t_{5}=59.5 ; t_{6}=74.3 ; t_{7}=89.2 ; t_{8}=96.7$. (b) The corresponding contamination profiles. (c) Path of the vortices (from time zero).
$W=1.90$ at $\operatorname{Re}=1680$, which would make the hole even smaller, and the agreement worse.

Although there are still considerable differences between the results for the two highest Reynolds numbers in the calculations in Figs. 7(c) and 7(d), the similarities are actually more striking than the differences. Furthermore, the changes between $\operatorname{Re}=1680$ and $\operatorname{Re}=3360$ are noticeably smaller than between 840 and 1680. In addition to that, the major differences are near the end of the runs. We are therefore tempted to make the conjecture that as the Reynolds number increases with $W$ constant, the solution becomes independent of $\operatorname{Re}$ for a time that becomes longer as the Reynolds number becomes larger. This is similar to what is observed for a number of other flows, and simply suggests that vorticity diffusion has not had time to modify the flow in a significant way. Finite viscosity is, of course, essential to 
balance the stresses at the surface created by the variation in surface tension. However, both the surface-tension-generated shear and the viscosity enter into $W$, and if viscosity is reduced, a "stronger" contaminant must be used to keep $W$ constant. The same argument applies to the circulation, $\Gamma$, which also appears in $W$. This conjecture will be the basis for our comparisons with the experiments of Hirsa and Willmarth. ${ }^{19}$

At late times, there will always be considerable dependency on the Reynolds number. In particular, the vortices are more long-lived the higher the Reynolds number. We have continued the $\operatorname{Re}=3360$ calculations up to a much longer time than the lower $\operatorname{Re}$ calculations and show selected frames, at late times, in Fig. 10 (a), as well as the corresponding contamination distribution in Fig. 10(b). The initial evolution is much like the run shown in Fig. 1(b); the primary vortex generates a secondary vortex that causes rebounding. Since the secondary vortex is much weaker than the primary vortex, the path curves inward as the secondary vortex is swung around the primary vortex eventually bringing both vortices back to the surface. The secondary vortex has now diffused considerably, but the primary one is still strong and causes a tertiary vortex to form. This tertiary vortex, although relatively weak, again leads to rebounding, and as the path of the vortices in Fig. 10(c) shows this pushes the primary vortex farther away from the surface than the first rebounding did. As the vortex pair comes back after the first rebound, the contamination shock is in the process of moving back inward. While the front of the shock continues to move inward, partly assisted by the secondary vortex, the primary vortex pushes the rest of the contaminant outward, thus creating the "hump" in the profile.

Long time calculation of the collision of a vortex pair with a rigid, no-slip wall have been reported by Orlandi. ${ }^{17}$ The vortices in his calculations loop back closer to the centerline than in Fig. 10, leading to an ejection of the secondary vortices for sufficiently high Reynolds numbers. The results in Figs. 1,2, and 4(a) suggest that similar behavior would be observed for higher $W$ 's (and the no-slip run) if the calculations were continued to later time.

\section{B. Comparison with experiments}

A detailed experimental study of the interaction of vortex pairs with a contaminated free surface has been done by Hirsa and Willmarth. ${ }^{19}$ They experimented with a vortex pair generated by a pair of flaps mounted in a water tank, and made a number of detailed measurements of the vortex motion and the flow field as well as the free surface signature. In addition to visualization by LIF, the velocity field was measured using particle image velocimetry. Extensive investigations of the three-dimensional evolution of a vortex pair colliding with a free surface were also done, but our two-dimensional calculations are only pertinent to the twodimensional aspects.

The calculations of the preceding section were for rather low Reynolds number and used a simple constitutive equation for the contaminants. Hirsa and Willmarth used exactly determined quantities of oleyl alcohol to vary the surface contaminant in a predetermined way and made detailed measurements of the surface tension for a given amount of surfactant. We have fitted an analytic expression to the measurements of Hirsa, ${ }^{19}$ and Fig. 11 shows the $c(\sigma)$ curve along with the experimentally determined points. The curve consists of three parts:

$$
\sigma(c)=\left\{\begin{array}{l}
70.838, \text { if } c<0.0828 \times 10^{-6} \\
45.08+62.22 c-37.575 c^{2} \\
\text { if } 0.0828 \times 10^{-6}<c<0.1242 \times 10^{-6} \\
103.03-31.11 c, \text { if } c>0.1242 \times 10^{-6}
\end{array}\right.
$$

Here $c$ is in units of $\mathrm{cm}^{3} / \mathrm{cm}^{2}$, and we have only fitted the low $c$ part of the relation, since $c$ never becomes large. Hirsa's measurements agree well with the data in Gaines. ${ }^{28}$ The Reynolds number of the experiments ranged from 11000 to 15600 , which would require excessive resolution, in particular, since our calculations were all done on a regular grid. However, as observed in Figs. 7 and 8 the evolution appears to be relatively insensitive to the Reynolds number once it is sufficiently high. We have therefore run a case comparable with the experimental setup, but kept the Reynolds number as well as the computational domain considerably smaller than in the experiments ( $R e=7000$, and a $256 \times 256$ grid). Here $W$ was, on the other hand, kept the same as in the experiments. The initial contamination concentration is $c_{0}=0.0848 \times 10^{-6} \mathrm{~cm}^{3} / \mathrm{cm}^{2}$. The initial conditions for the vorticity came from the experimentally determined velocity field. The results are shown in Fig. 12, where the vortex path, as well as the extend of the clean region above the vortices, is compared with the experimental results.

There are some differences. The maximum "opening" of the contaminant layer is smaller in the computations than in the experiments, and as a consequence, the secondary vortex forms closer to the centerline, and the primary vortex re-

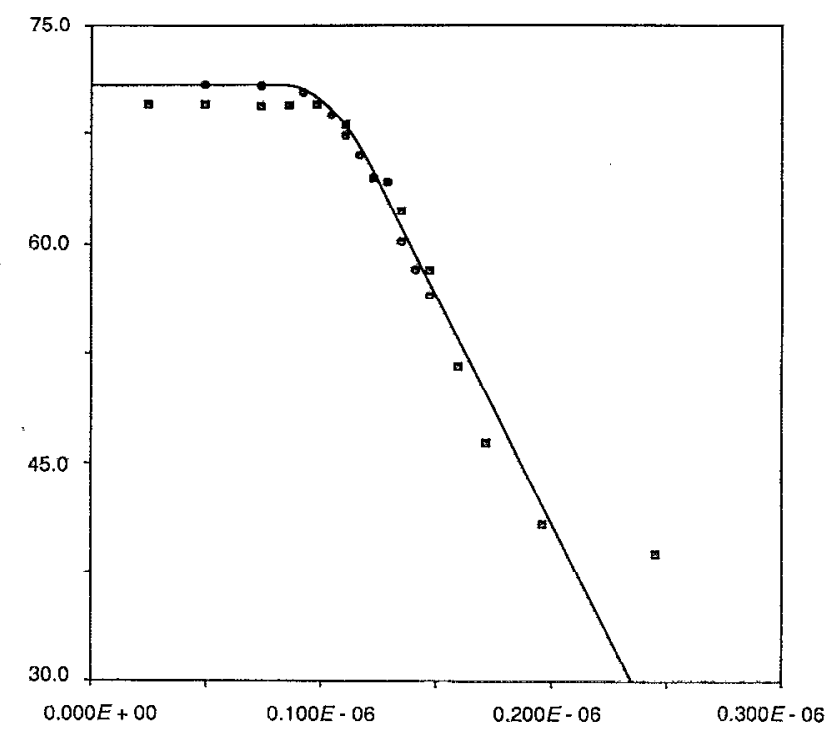

FIG. 11. Comparison with experiments, analytical fit to the $\sigma(c)$ curve and the measurements (squares and circles) of Hirsa et al. The initial contaminant concentration is $0.0843 \times 10^{-6} \mathrm{~cm}^{3} / \mathrm{cm}^{2}$. 


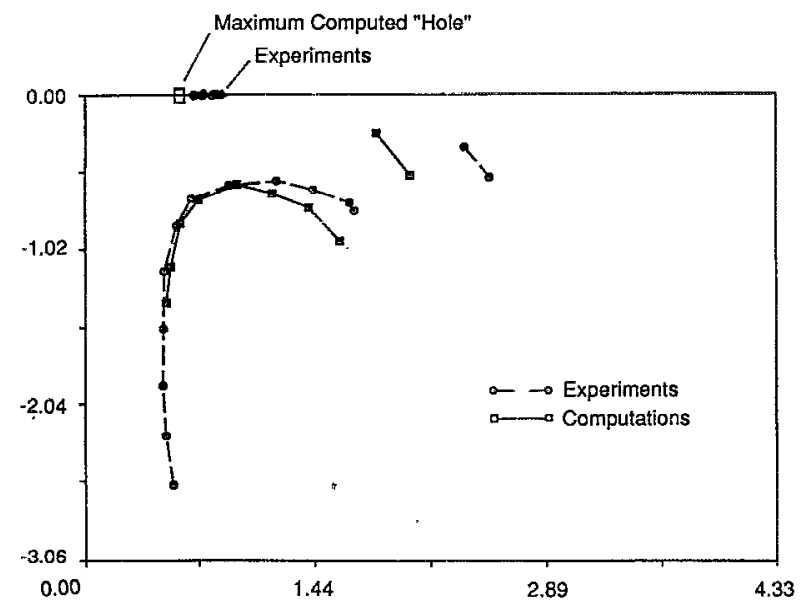

FIG. 12. Comparison with experiments, vortex paths, and the extent of the clean "hole" in the contaminant layer.

bounds more. Apart form the obvious differences in Reynolds number, small errors in the constitutive equation may also be responsible for the deviations. In addition to $\mathrm{Eq}$. (9), we have also used slightly different analytical fits for the contaminant equation of state, and find considerable sensitivity due to the steepness of the curve. It is therefore likely that a minor change in the equation of state would lead to a better agreement. A careful examination of Fig. 11 suggest that a slightly smaller slope, around $c=0.1 \times 10^{-6}$, would be consistent with the experimental data. Such a change would lead to changes in the right direction. We have elected to present these results, nevertheless, because we feel that they represent the level of agreement that may be expected without any "tuning" of the fit. The agreement, although not perfect, certainly suggest that the experimentally observed behavior is mainly due to the contaminant and not due to, say, surface deformations.

\section{Axisymmetric vortex rings}

The main focus in this paper is on two-dimensional vortex pairs. As discussed by Bernal et al. ${ }^{18}$ axisymmetric vortex rings are similarly affected by contaminants on the free surface. The major difference from the planar case is the stretching of the vortex ring as it moves outward along the top boundary. This stretching opposes the increase in the core size by diffusion and does lead to vorticity intensification for high enough Reynolds numbers.

We have done a few calculations for axisymmetric rings, and Fig. 13 shows two examples. In (a) the top surface is stress-free and in (b) $W=3.6$. The Reynolds number is 2000 in both cases, and the computational box is relatively small. The evolution is similar to the two-dimensional case; in the simulation with stress-free boundaries the vortex moves outward to the outer boundaries but rebounds when the surface is contaminated. Since the vortex ring expands as it moves outward, diffusion of vorticity is countered by vorticity intensification due to stretching. For the stress-free sur- (a)

(b)

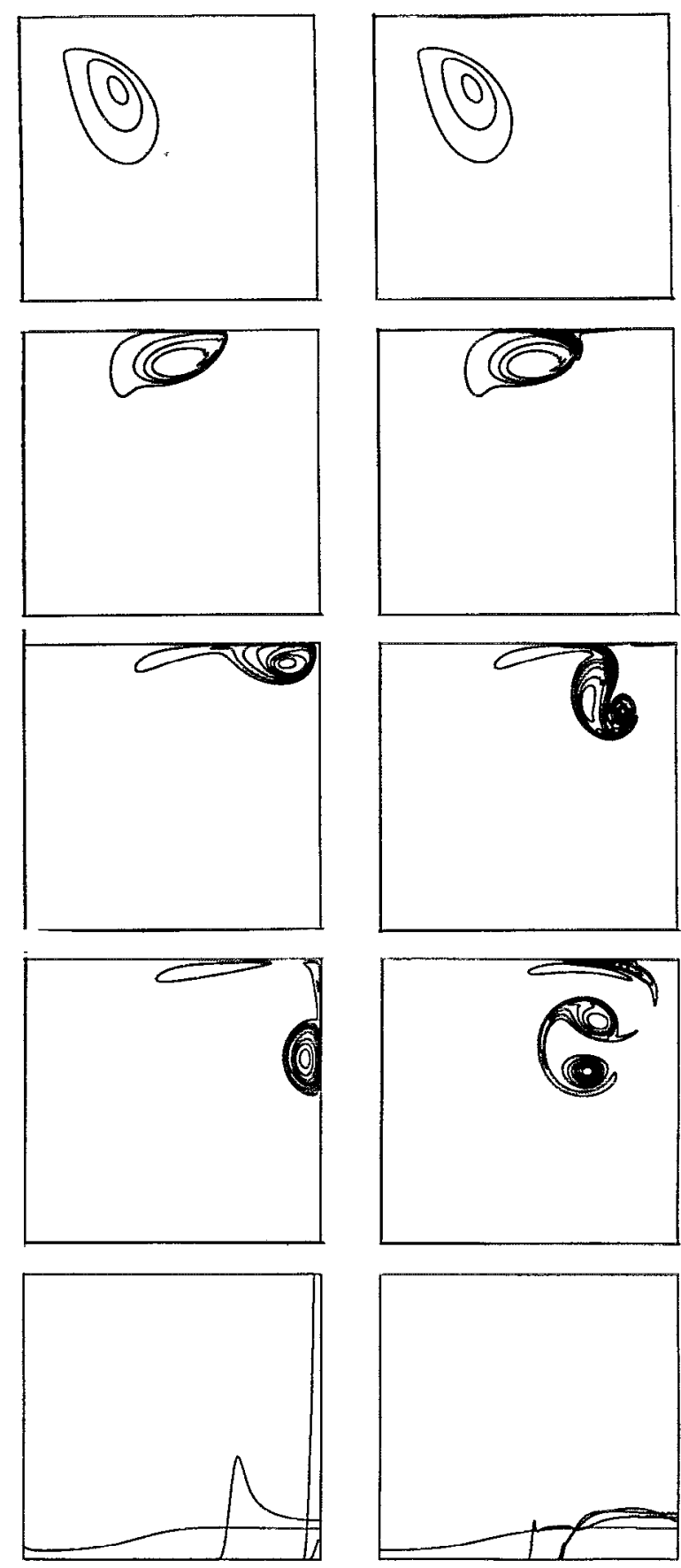

FIG. 13. The evolution of an axisymmetric vortex ring with (a) clean surface and (b) contaminated surface. The vorticity is shown at selected times, and the corresponding contamination profiles in the bottom frames.

face our calculations are in good agreement with those of Orlandi. ${ }^{17}$

\section{Oblique collisions of vortex pairs}

All the computations discussed so far involve a vortex pair colliding head on with the surface. To show that the 
basic interaction mechanisms are insensitive to the exact angle of approach, we have run one case where the vortices approach the surface at a $45^{\circ}$ angle. The results are shown in Fig. 14. Here $\mathrm{Re}=1680$ and $W=0.95$. Initially the top vortex has more influence on the contaminant distribution, and the contaminant is mostly pushed to the right. When this vortex is sufficiently close to the surface it starts to move backward (to the right) due to the effect of its image, and in the process scoops up vorticity from the boundary layer created by the uneven contaminant distribution. It then rebounds. The second vortex has now come closer to the surface- so the vortex pair is actually facing the surface more directly-and pulls vorticity from the boundary layer. This vortex does not make it as close to the surface as the first one, since its partner has left it, and the secondary vortex is much weaker than the partner. Consequently, the rebound of the second vortex is much smaller than for the first one. While the second vortex continues to move slowly to the left, the first vortex has swept its secondary vortex around itself, as well as moved in a small loop. It therefore collides with the surface again pulling out a tertiary vortex. The secondary vortex has in the mean time diffused away and reduced the circulation of the primary one. We are now left with essentially one vortex that moves slowly down and to the left. The contamination shock on the right moves back in to close the hole, but on the left, the shock is nearly stationary in the last frame. A careful examination of the location of the right shock shows that the looping of the primary vortex is reflected in slight oscillation of the location of the shock.

This example suggests that the behavior observed in the

(a)
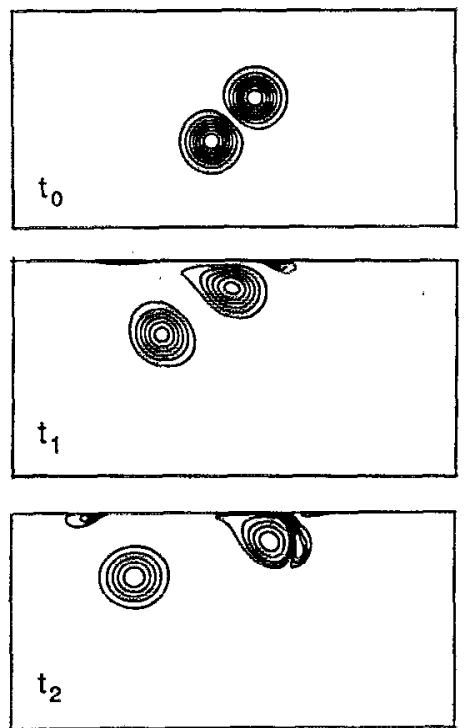

(b)
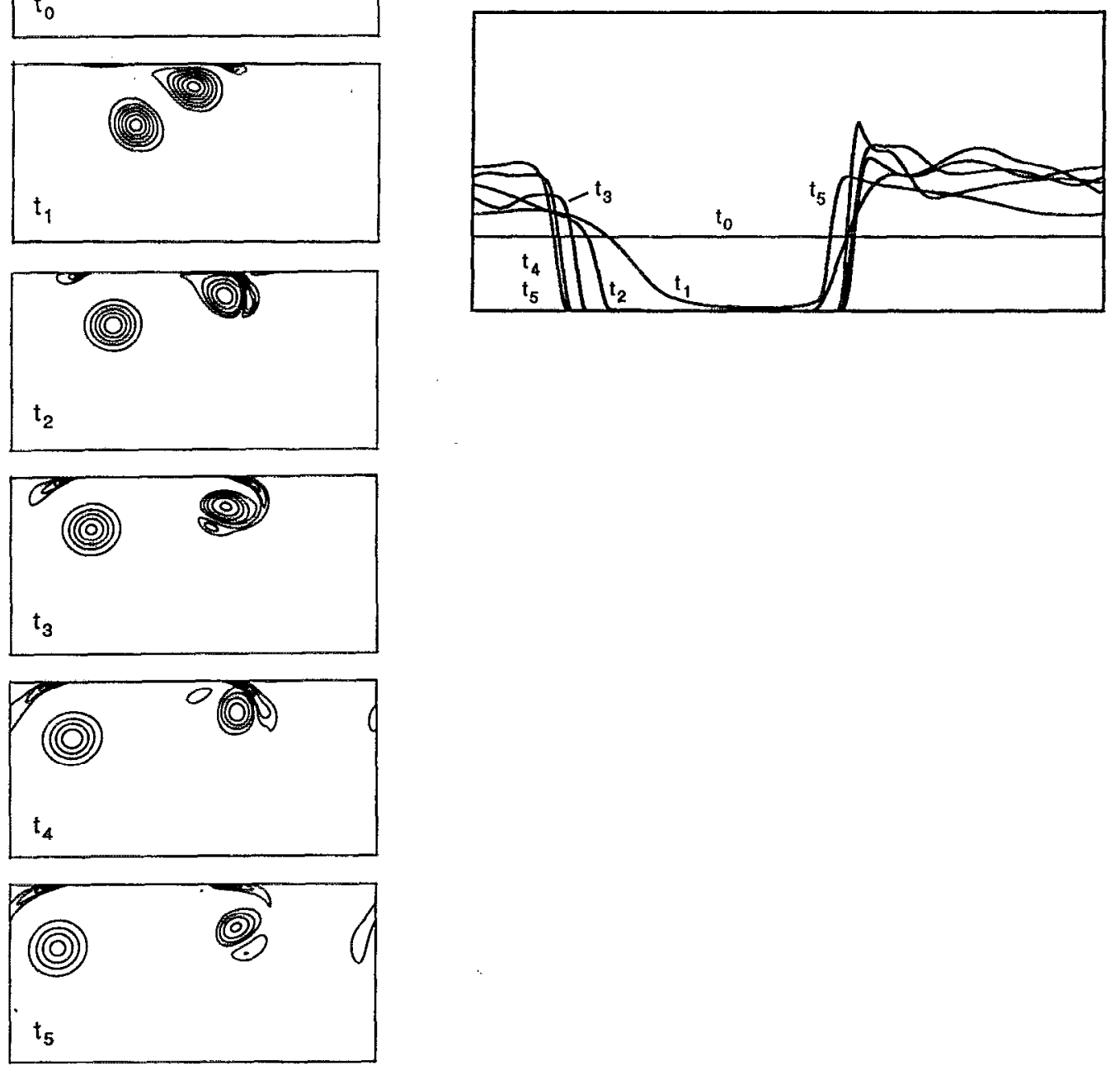

FIG. 14. Oblique collision of a vortex pair with a contaminated surface. The vorticity is shown at selected times in (a) and the corresponding contamination profiles in (b). Here, periodic boundary conditions are used in the horizontal direction. 
head on collisions studied earlier in the paper is actually stable to variations in the approach angle. Although there are some differences for the rather extreme case of $45^{\circ}$, these are of a relatively obvious nature.

\section{CONCLUSIONS}

We have investigated in some detail the influence of free surface contamination on the motion of a vortex pair toward a waveless free surface. Several two-dimensional simulations for various values of the governing nondimensional numbers, $\operatorname{Re}$ and $W$, have been done. The main observations of the present work are a confirmation of the experimental results of Bernal et al. ${ }^{18}$ and Hirsa and Willmarth, ${ }^{19}$ that contamination of the free surface can alter the submerged vortical motion in a fundamental way. This alteration-for the kind of flow considered here-is primarily through injection or separation of vorticity created at the surface by uneven surface tension. Since a very simple equation of state for the contaminant lead to similar results as a more realistic constitutive equation (and experiments) it is likely that all surfactants affect submerged vortical flow in this way.

Although our calculations have been limited to a somewhat low Reynolds number, the results suggest that at early times and high enough Reynolds numbers the evolution depends primarily on $W$ and only weakly on Re. For low $W$, the surface contaminant is redistributed considerably by the flow, but for higher $W$ the surface resists the motion, acting more or less like a rigid wall in the limit of very high $W$. Intermediate values results in localized clean regions, separated from the contaminated surface by a sharp "shock." Of course, for long enough time, $\mathrm{Re}$ will eventually determine the rate of decay of the vorticity. For all $\operatorname{Re}$ that we have simulated, the addition of contaminant greatly increases the rate of decay of the circulation of the primary vortex. Generally, shortly after generation the secondary vortices have strength about one-fourth to one-fifth of the primary vortex, which is consistent with the single measurement taken by Hirsa and Willmarth. ${ }^{19}$ For large enough $W$, the contaminant distribution remains almost flat for all time, and the evolution of the vortical flow is nearly indistinguishable from the no-slip case. Therefore, at high Re and $W$ we expect the evolution to be independent of the actual value of these parameters.

The major limitation of this study is that the free surface has been taken as tlat. This limits the applicability of the predictions to small Froude numbers, but the comparison with the experiments of Hirsa and Willmarth, ${ }^{19}$ which were conducted at low Froude numbers, suggests that this approximation does not bias the results in any significant way for these Froude numbers.

Although the main conclusion from this study is a conformation of the mechanism already explained experimentally by Bernal et al. ${ }^{18}$ and Hirsa and Willmarth, ${ }^{19}$ we note that the flexibility of the numerical approach has allowed us to obtain information that is extremely difficult, expensive, and time consuming to measure (e.g., how the distribution of the surface contaminant changes with time), and to explore parameter combinations difficult to realize experimentally [e.g., the shape and magnitude of $\sigma(c)$ ].

\section{ACKNOWLEDGMENTS}

Constructive interaction with Professor L. Bernal and Professor W. J. A. Dahm and other members of the PSH has been most helpful in carrying out the research discussed here. G. T. would like to thank P. Orlandi for discussions and for sending preprints of this work.

This work was supported by the Program in Ship Hydrodynamics (PSH) at the University of Michigan, funded by the University Research Initiative of the Office of Naval Research (Contract No. N000184-86-K-0684). The calculations were done mostly on the computers at the San Diego Supercomputer Center, which is sponsored by the National Science Foundation.

${ }^{1}$ T. Sarpkaya, "Trailing-vortex wakes on the free surface," 16th Symposium on Naval Hydrodynamics (National Academy Press, Washington, DC, 1986), pp. 38-50.

${ }^{2}$ W. W. Willmarth, G. Tryggvason, A. Hirsa, and D. Yu, "Vortex pair generation and interaction with a free surface," Phys. Fluids A 1, 170 (1989).

${ }^{3}$ T. Sarpkaya, J. Elnitsky, II, and R. E. Leeker, Jr., "Wake of a vortex pair on the free surface,". 17th Symposium on Naval Hydrodynamics (National Academy Press, Washington, DC, 1989), pp. 53-60.

${ }^{4} \mathrm{~L}$. P. Bernal and K. Madnia, "Interaction of a turbulent round jet with the free surface," in Proceedings of 17th Symposium on Naval Hydrodynamics (National Academy Press, Washington, DC, 1989), pp. 79-87.

${ }^{5}$ L. P. Bernal and J. T. Kwon, "Vortex ring dynamics at a free surface," Phys. Fluids A 1, 449 (1989).

${ }^{\circ} \mathrm{M}$. Song, L. Bernal, and G. Tryggvason, "Head-on collision of a large yortex ring with a free surface," to appear in Phys. Fluids A.

${ }^{7} \mathrm{G}$. Tryggvason, "Deformation of a free surface as a result of vortical flows," Phys. Fluids 31, 955 (1988).

${ }^{8} \mathrm{~J}$. H. Telste, "Potential How about two counter-rotating vortices approaching a free surface," J. Fluid Mech. 201, 259 (1989).

${ }^{\circ}$ D. L. Marcus and S. A. Berger, "The interaction between a pair of counter-rotating vortex pairs in vertical ascent and a free surface," Phys. Fluids A 1, 1988 (1989).

${ }^{10} \mathrm{D}$. Yu and G. Tryggvason, "The free surface signature of unsteady, twodimensional vortex flows," J. Fluid Mech. 218, 547 (1990).

" J. T. Davies, "The effects of surface films in damping eddies at a free surface of a turbulent liquid," Proc. R. Phys. Soc. A 290, 515 (1966).

${ }^{12}$ J. T. Davies and J. P. Driscoll, "Eddies at free surfaces, simulated by pulses of water," Ind. Eng. Chem. Fundamen. 13, 105 (1974).

${ }^{13}$ S. J. Barker and S. C. Crow, "The motion of two-dimensional vortex pairs in a ground effect," J. Fluid Mech. 82, 659 (1977).

${ }^{14} \mathrm{~J}$. K. Harvey and F. J. Perry, "Flowfield produced by trailing vortices in the vicinity of the ground," AIAA J. 9, 1659 (1971).

${ }^{15} \mathrm{P}$. G. Saffman, "The approach of a vortex pair to a plane surface in inviscid fiuid," J. Fluid. Mech. 92, 497 (1979).

${ }^{16}$ A. J. Peace and N. Riley, "A viscous vortex pair in ground effect," J. Fluid Mech. 129, 409 (1983).

${ }^{17}$ P. Orlandi, "Vortex dipole rebound from a wall," Phys. Fluids A 2, 1429 (1990); P. Orlandi, "Vortices interacting with no-slip walls: Stirring and mixing of primary and secondary vorticity," Phys. Fluids A 3, 1463 (1991).

${ }^{18}$ L. P. Bernal, A. Hirsa, J. T. Kwon, and W. W. Wilmarth, "On the interaction of vortex rings and pairs with a free surface for various amounts of a surface active agent," Phys. Fluids A 1, 2001 (1989).

${ }^{19} \mathrm{~A}$. Hirsa and W. W. Willmarth, "Measurements of vortex pair interaction with a clean and contaminated free surface," submitted to J. Fluid Mech.; A. Hirsa, "An experimental investigation of vortex pair interaction with a clean or contaminated free surface," Ph. D. thesis, The University of Michigan, 1990.

${ }^{20} \mathrm{~J}$. C. Scott, "Flow beneath a stagnant film on water: The Reynolds ridge," J. Fluid Mech. 116, 283 (1982).

${ }^{21} \mathrm{~J}$. F. Ilarper, "The motion of bubbles and drops through liquids," Adv. Appl. Mech. 12, 59 (1972). 
${ }^{22} \mathrm{H}$. A. Stone and L. G. Leal, "The effects of surfactants on drop deformation and breakup," J. Fluid Mech. 220, 161 (1990).

${ }^{23}$ S. Ostrach, "Low-gravity fluid flows," Annu. Rev. Fluid Mech. 14, 313 (1982).

${ }^{24}$ W. J. A. Dahm, C. M. Scheil, and G. Tryggvason, "Dynamics of vortex interaction with a density interface," J. Fluid Mech. 205, 1 (1989).

${ }^{25} \mathrm{~S}$. Ohring and H. J. Lugt, "Interaction of a viscous vortex pair with a free surface," J. Fluid Mech. 227, 47 (1990); "Two counter-rotating vortices approaching a free surface in a viscous fluid," David Taylor Research Center Report No. DTRC-80/019, Bethesda, MD, 1989.
${ }^{20} \mathrm{H}$. T. Wang and R. T. Leighton, "Direct calculation of the interaction between subsurface vortices and surface contaminants," proceedings of the 9th OMAE Conference, Houston, TX, Feb., 1990 (ASME, New York, 1990), Vol. I, Part A.

${ }^{27}$ A. Hirsa, G. Tryggvason, J. Abdollahi-Alibeik, and W. W. Willmarth, "Measurement and computations of vortex pair interaction with a clean or contaminated free surface," in Proceedings of 18th Symposium on $\mathrm{Na}$ val Hydrodynamics (National Academy Press, Washington, DC, 1990).

${ }^{28} \mathrm{G}$. L. Gaines, Jr., Insoluble Monolayers at Liquid-Gas Interface (Interscience, New York, 1966). 